\title{
Shared signaling networks active in B cells isolated from genetically distinct mouse models of lupus
}

\author{
Tianfu Wu, Xiangmei Qin, Zoran Kurepa, Kirthi Raman Kumar, Kui Liu, Hasna Kanta, Xin J. Zhou, \\ Anne B. Satterthwaite, Laurie S. Davis, and Chandra Mohan
}

Division of Rheumatology, Department of Internal Medicine, University of Texas Southwestern Medical Center, Dallas, Texas, USA.

\begin{abstract}
Though B cells play key roles in lupus pathogenesis, the molecular circuitry and its dysregulation in these cells as disease evolves remain poorly understood. To address this, a comprehensive scan of multiple signaling axes using multiplexed Western blotting was undertaken in several different murine lupus strains. PI3K/AKT/ mTOR (mTOR, mammalian target of rapamycin), MEK1/Erk1/2, p38, NF- $\mathrm{BB}$, multiple Bcl-2 family members, and cell-cycle molecules were observed to be hyperexpressed in lupus $B$ cells in an age-dependent and lupus susceptibility gene-dose-dependent manner. Therapeutic targeting of the AKT/mTOR axis using a rapamycin (sirolimus) derivative ameliorated the serological, cellular, and pathological phenotypes associated with lupus. Surprisingly, the targeting of this axis was associated with the crippling of several other signaling axes. These studies reveal that lupus pathogenesis is contingent upon the activation of an elaborate network of signaling cascades that is shared among genetically distinct mouse models and raise hope that targeting pivotal nodes in these networks may offer therapeutic benefit.
\end{abstract}

\section{Introduction}

SLE is a chronic, multisystem autoimmune disease associated with the production of a myriad of autoantibodies, primarily against nuclear antigens (1). Some of these antibodies may be responsible for tissue damage in this disease. An increasing body of data demonstrates that intrinsic hyperactivity of B cells might represent a key phenomenon in the development of lupus, both in mice and humans (2-6). However, the underlying molecular mechanisms have not been fully elucidated. Work in several laboratories has revealed that the genetic manipulation of several signaling axes can apparently precipitate lupus-like disease. For instance, forced hyperexpression of PI3K (7) or the antiapoptotic molecule Bcl-2 (8) as well as haploinsufficiency of the tumor suppressor phosphatase and tensin homolog (PTEN) $\left(\right.$ Pten $\left.^{+/-}\right)(9)$ have been shown to cause lymphoproliferative lupus. Although the above studies have uncovered various signaling axes that can potentially have an impact on lupus in "synthetic" models, the signaling pathways that are aberrantly upregulated in spontaneously arising lupus remain a black box. The goal of this study is to execute a comprehensive analysis of the signaling axes that become dysregulated in murine lupus, particularly with disease evolution.

Lupus has been extensively studied in several murine models, including BWF, NZM2410, BXSB, and MRL/lpr mice (10-12). Importantly, in most of these models, it has been demonstrated that the B cells are intrinsically abnormal and absolutely essential for development of the disease (13-15). Nevertheless, the molecular cascades that mediate $\mathrm{B}$ cell hyperactivity in these models remain

Nonstandard abbreviations used: $\mathrm{BCR}, \mathrm{B}$ cell receptor; $\mathrm{CDK}$, cyclin-dependent

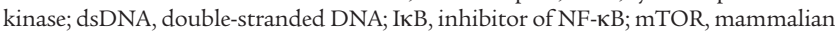
target of rapamycin; mTORC, mTOR complex; PTEN, phosphatase and tensin homo$\log$; Rb, retinoblastoma protein; Sle1, SLE susceptibility locus 1.

Conflict of interest: The authors have declared that no conflict of interest exists. Citation for this article: J. Clin. Invest. 117:2186-2196 (2007). doi:10.1172/JCI30398. poorly understood. Over the past decade, we have learned more about the genetic basis of lupus in these models (16-18). In particular, we have learned that the development of lupus in the NZM2410 strain requires the presence of 3 chromosomal intervals referred to as SLE susceptibility locus 1 (Sle1), Sle2, and Sle3 (19). To further study the importance of each interval in the development of SLE, the NZM2410-derived $z$ alleles of these loci have been introgressed onto the lupus resistant C57BL/6 (B6) background as congenic strains. Interestingly, these different loci were associated with very different component lupus phenotypes (17). The presence of the $\mathrm{z}$ allele of the Sle1 interval on the B6 background in the B6.Sle1 strain was sufficient to break immune tolerance to chromatin, resulting in serological autoreactivity to histone/DNA complexes (20). However, for the development of full-blown lymphoproliferative lupus marked by anti-double-stranded DNA (anti-dsDNA) antibodies, nephritis, lymphadenopathy, splenomegaly, and mortality, an epistatic interaction of Sle1 with other loci, such as Yaa, Sle3, FASlpr, etc., was apparently required $(19,21,22)$.

Of particular relevance to this communication are the 2 congenic strains B6.Sle1 and B6.Sle1.Sle3. Compared with B6 controls, B6.Sle1 mice exhibit incipient autoimmunity, as described above. Importantly, it is known that Sle1 functions in a B cell-intrinsic fashion (23). In contrast, B6.Sle1.Sle3 bicongenic mice develop fullblown lupus nephritis (21). Though the NZM2410-derived $z$ allele of Sle 3 has a profound impact on myeloid cells (24), it also appears to affect $B$ cells in an intrinsic fashion (25). By taking advantage of the fact that the B6.Sle1 and B6.Sle1.Sle 3 congenic strains capture 2 distinct stages of severity in the evolution of lupus and the fact that both loci have an impact on B cell function, we set out to obtain a comprehensive view of the signaling pathways that become activated in B cells as lupus evolves, using these novel genetic models. Furthermore, by establishing the activation status of different signaling axes in lupus, one can potentially identify better targets for therapeutic intervention in this disease. We demonstrate that 


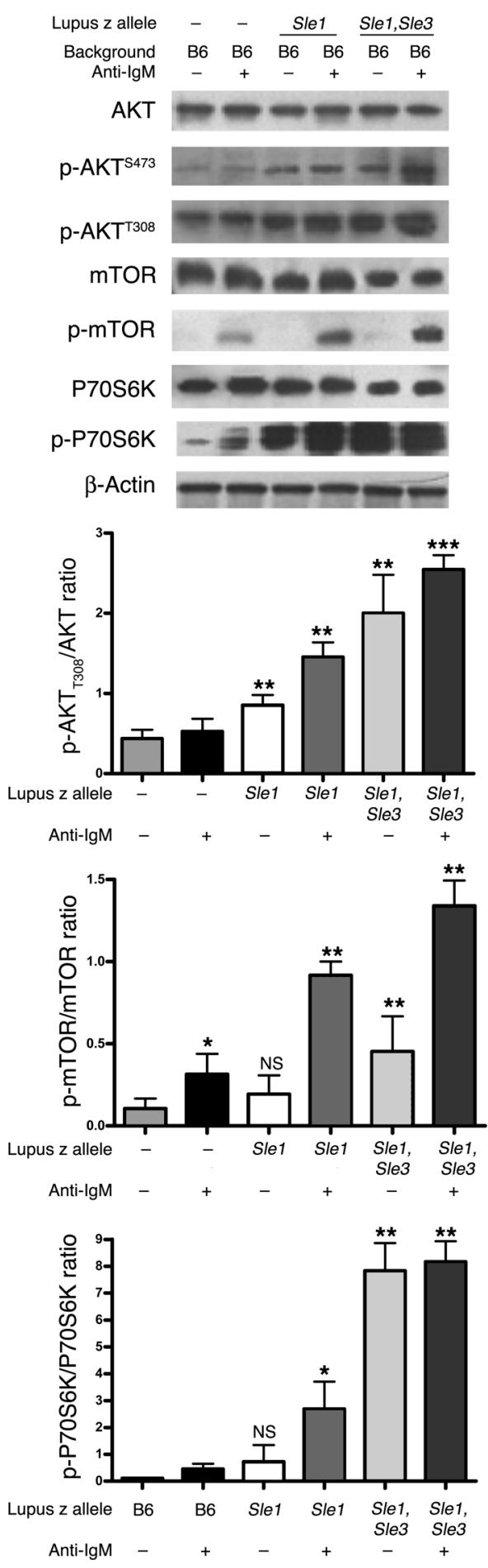

the use of the mammalian target of rapamycin (mTOR) inhibitor RAD001 (everolimus) ameliorates the development of disease in these congenic models of lupus. Surprisingly, this therapeutic benefit was associated with the dampening of multiple signaling axes in addition to the AKT/mTOR pathway.

\section{Results}

To uncover the signaling axes that may be upregulated in lupus lymphocytes, 2 different Western blot-based screening exercises

\section{Figure 1}

Activation status of AKT/mTOR axis in lupus B cells. Anti-B220 beadpurified splenic B cells were isolated from 2-month-old female B6, B6.Sle 1z, and B6.Sle1z.Sle3z mice, anti-IgM F $\left(a b^{\prime}\right)_{2}$ stimulated, lysed, electrophoresed, and blotted using a panel of antibodies specific for various molecules in the AKT/mTOR axis, some of which were specific for the phosphorylated forms of the signaling molecules. The mean $( \pm$ SEM) values of 3-6 individual samples (derived from independent mice) are plotted on the right The $P$ values shown pertain to a Student's $t$ test comparison of the 24-hour-stimulated (or unstimulated) congenic samples with the corresponding stimulated (or unstimulated) B6 samples, respectively. ${ }^{\star} P<0.05 ;{ }^{* \star} P<0.01$; ${ }^{* \star} P<0.001$.

were initially undertaken through commercial services. In the first screening exercise, splenocytes isolated from gender-matched 6-month-old B6.Sle $1^{\mathrm{z}}$, B6.Sle ${ }^{\mathrm{z}}$.Sle $3^{\mathrm{z}}$, and B6 controls were scanned by Kinexus using KPKS-1.1 and KPSS- 4.1 screening platforms for the expression of several different signaling molecules. Screening blots suggested that multiple signaling axes may be upregulated in lupus splenocytes, including the PI3K/AKT/mTOR axis, p38, the Ras/MEK1/Erk1/2 axis, the NF-KB pathway, several Bcl-2 family members, and JAK/STAT molecules as well as cell-cycle proteins (data not shown). In a second screening experiment, we examined the status of signaling pathways in splenic B cells obtained from 2-month-old B6 and B6.Sle $1^{\mathrm{z}}$ mice. Using the BD PowerBlot screening service, we scanned unstimulated or anti-IgM F $\left(\mathrm{ab}^{\prime}\right)_{2}$-stimulated purified B cells for the expression levels of a large number of molecules. BD PowerBlot scanning revealed additional signaling molecules to be upregulated in B6.Sle $1^{z} \mathrm{~B}$ cells, including MEK2 and STAT1 (data not shown).

Importantly, these initial screening exercises revealed that $\mathrm{B}$ cell activation in lupus may not be restricted to isolated signaling cascades - indeed, almost all of the signaling pathways examined seemed to be dysregulated in lupus B cells. Since commercial screening services preclude feasibility of multiple replicates and repeat assays, we proceeded to examine these different axes by Western blotting in a more systematic fashion using larger numbers of unstimulated and stimulated B cells isolated from B6, B6.Sle ${ }^{z}$, and B6.Sle $1^{z}$.Sle ${ }^{z}$ spleens. For these studies, we used mice at 2 to 3 months of age, well before any disease manifestations. At the age mice were examined, these 3 strains did not differ in their relative percentages of follicular versus marginal zone B cells in their spleens (data not shown).

We first examined the PI3K/AKT/mTOR axis. Compared with the B6 controls, unstimulated B6.Sle $1^{\mathrm{z}}$ and B6.Sle ${ }^{\mathrm{z}}$.Sle $3^{\mathrm{z}} \mathrm{B}$ cells exhibited significantly higher levels of AKT phosphorylation (Figure 1). Levels of AKT activation were greatest in B6.Sle $1^{z}$.Sle $3^{z} \mathrm{~B}$ cells. Moreover, phosphorylation at the threonine residue T308 in the catalytic domain of AKT was more pronounced than phosphorylation at the serine residue S473 in the carboxyterminal hydrophobic motif. We also saw a parallel increase in the phosphorylation of several downstream targets of AKT, including mTOR and P70S6K (Figure 1). In particular, B6.Sle $1^{\mathrm{z}}$ and $\mathrm{B} 6$. Sle $^{\mathrm{z}}{ }^{\mathrm{z}}$.Sle $3^{\mathrm{z}} \mathrm{B}$ cells exhibited severalfold more phosphorylated P70S6K, even when mice were 2 months of age (Figure 1). Further upregulation of this signaling axis was achieved by cross-linking the B cell receptor (BCR), as evidenced by the increased phosphorylation of $\mathrm{AKT}^{\mathrm{T} 308}$, mTOR, and P70S6K compared with that in BCR-cross-linked B6 B cells (Figure 1).

A second signaling pathway found to be upregulated in lupus B cells through the original screening assays was the Ras/Raf/MEK1/ 
Erk $1 / 2$ axis. This was confirmed by Western blot analysis, which showed that unmanipulated 2-month-old B6.Sle $1^{z}$.Sle $3^{z}$ B cells expressed significantly higher levels of phosphorylated MEK1/2 and Erk $1 / 2$, with intermediate levels being noted in B6.Sle $1^{\mathrm{z}} \mathrm{B}$ cells in comparison with B6 controls (Figure 2). As noted with the AKT/ $\mathrm{mTOR}$ axis, $\mathrm{B}$ cells from the congenic strains were more sensitive to BCR cross-linking since they exhibited significantly higher levels of $\mathrm{p}-\mathrm{MEK} 1 / 2$ and $\mathrm{p}$-Erk1/2 upon stimulation than did B6 $\mathrm{B}$ cells. Levels of $\mathrm{p} 38^{\mathrm{MAPK}}$ were also elevated in B6.Sle ${ }^{\mathrm{z}}$.Sle ${ }^{\mathrm{z}} \mathrm{B}$ cells, but the level of another key MAPK molecule, JNK, was unaltered (Figure 2 and data not shown). A third axis that was significantly

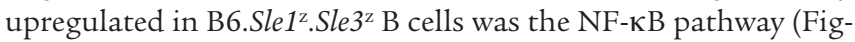

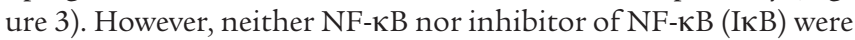
upregulated or phosphorylated in unmanipulated B6.Sle ${ }^{z} \mathrm{~B}$ cells, indicating that epistatic interaction with Sle 3 was essential for the spontaneous upregulation of this axis.

Several members of the Bcl-2 family have previously been reported to be aberrantly expressed in lupus B cells (26-28). Levels of the antiapoptotic molecules $\mathrm{Bcl}-2$ and $\mathrm{Bcl}-\mathrm{xl}$ and the proapoptotic molecules Bim and Bax were assayed in unmanipulated or stimulated splenic $\mathrm{B}$ cells from B6.Sle $1^{\mathrm{z}}$ and B6.Sle ${ }^{\mathrm{z}}$.Sle $3^{\mathrm{z}}$ congenics. In both cases, ratios of antiapoptotic to proapoptotic molecules were significantly elevated in lupus B cells (Figure 4). In B6.Sle $1^{\mathrm{z}} \mathrm{B}$ cells, this was largely due to the significant downregulation of Bim and Bax, particularly the latter. In contrast, in B6.Sle $1^{\mathrm{z}}$.Sle $3^{\mathrm{z}} \mathrm{B}$ cells, both upregulation of the antiapoptotic molecules (Bcl-2 in particular) and downregulation of Bim and Bax appeared to be contributing. These differences in expression became even more skewed upon BCR ligation, largely due to the more exaggerated loss of Bax in Sle 1z-bearing B cells (Figure 4). Interestingly, the level of $\mathrm{p}$-Bad was significantly upregulated in B6.Sle ${ }^{\mathrm{z}} \mathrm{B}$ cells but downregulated in B6.Sle ${ }^{\mathrm{z}}$.Sle $3^{\mathrm{z}} \mathrm{B}$ cells (Figure 4).

To assess how the upregulation of these signaling pathways might have an impact on cell cycling, we evaluated the status of various molecules involved in cell cycling. As one might have predicted from the above signaling profiles, B6.Sle $1^{\mathrm{z}}$ and B6.Sle ${ }^{\mathrm{z}}$.Sle ${ }^{\mathrm{z}}$ $B$ cells exhibited significant downregulation of $\mathrm{p}^{2} 7^{\mathrm{Kip} 1}$ and significant upregulation of the G1-phase and S-phase cyclin/cyclindependent kinases (CDKs) (Figure 5). In particular, unmanipulated B cells from 2-month-old B6.Sle $1^{\mathrm{z}}$ mice exhibited robust elevations in the levels of CDK2 and cyclin D3, with these elevations becoming even more pronounced in B6.Sle $1^{\mathrm{z}}$.Sle $3^{\mathrm{z}} \mathrm{B}$ cells (Figure 5). There were, however, no significant strain differences following anti-IgM stimulation. In addition, lupus B cells exhibited increased phosphorylation of retinoblastoma protein ( $\mathrm{Rb}$ ) and significantly decreased phosphorylation of the transcription factor E2F. All of these distal signaling events were only modestly affected by BCR cross-linking in vitro (Figure 5).

Several components of the JAK/STAT axis were also upregulated in lupus B cells. Particularly striking was the significant upregulation of phosphorylated JAK2 and STAT5 in unmanipulated Sle $1^{2}$-bearing B cells from 2-month-old mice (Figure 6). p-STAT3 was not significantly increased in B6.Sle $1^{z}$ B cells but became profoundly upregulated in B cells from the bicongenic strain (Figure 6). No additional differences were noticed following BCR stimulation. In contrast to most of the signaling axes $/ \mathrm{mol}-$ ecules that were upregulated in lupus B cells, several PKC isoforms were significantly downregulated in lupus B cells, in particular, PKC $\alpha$, PKCס, and PKCE (Figure 6 and data not shown).

We next examined the status of these signaling pathways as a function of age. The aforementioned signaling axes were evalu-
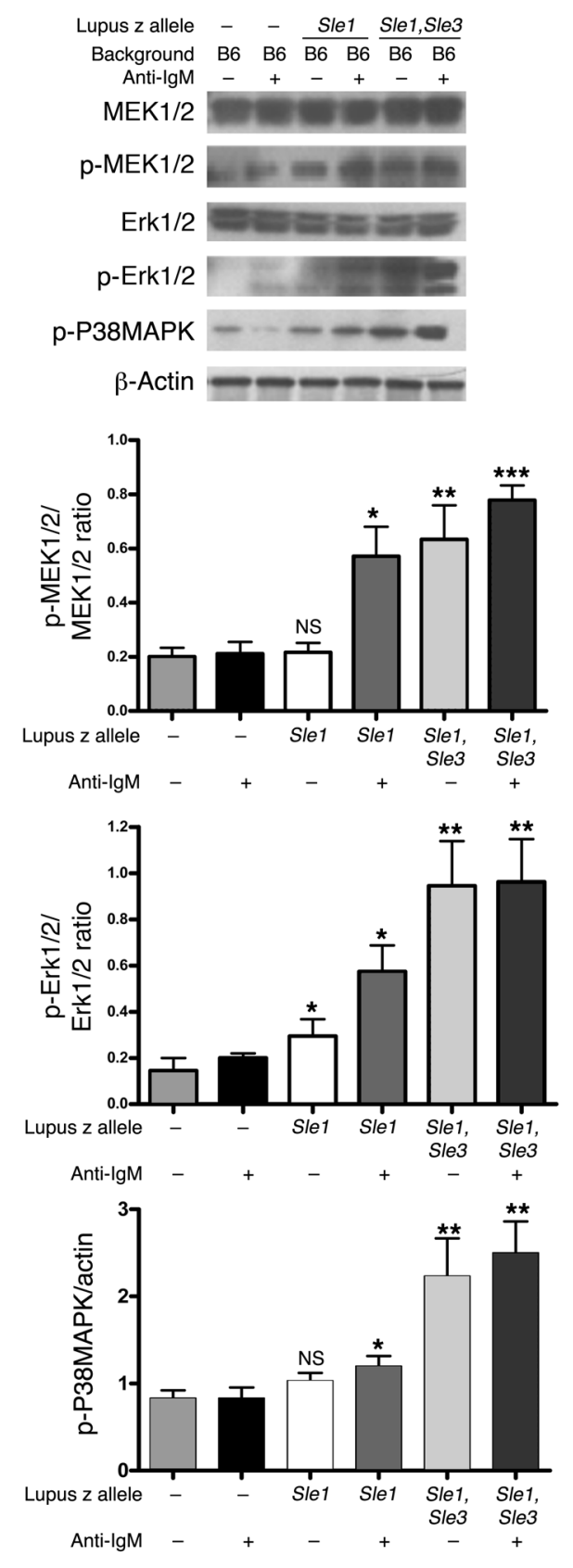

Figure 2

Activation status of Ras/MEK1/Erk1/2 pathway in lupus B cells. Legend is as noted for Figure 1, except that various molecules in the Ras/MEK $1 /$ Erk $1 / 2$ axis were specifically examined. ${ }^{*} P<0.05$; ${ }^{\star \star} P<0.01 ;{ }^{* \star} P<0.001$.

ated in splenic B cells isolated from 2-, 4-, and 6-month-old B6.Sle $1^{z}$.Sle ${ }^{z}$ congenics. These bicongenics are known to be disease free at 2 months of age but severely diseased at 6 months (ref. 21 and data not shown). As shown in Table 1, all of the differentially expressed pathways described above become progressively upregulated with age. Most of the signaling molecules were significantly elevated in 4-month-old mice compared with 2-month-old mice. Phosphorylation levels of p-AKT, p-mTOR, p-Bcl-2, p-IкB $\alpha$, $\mathrm{p}-\mathrm{NF}-\mathrm{\kappa B}, \mathrm{p}$-Erk1/2, and $\mathrm{p}$-STAT3 were even higher in 6-month- 

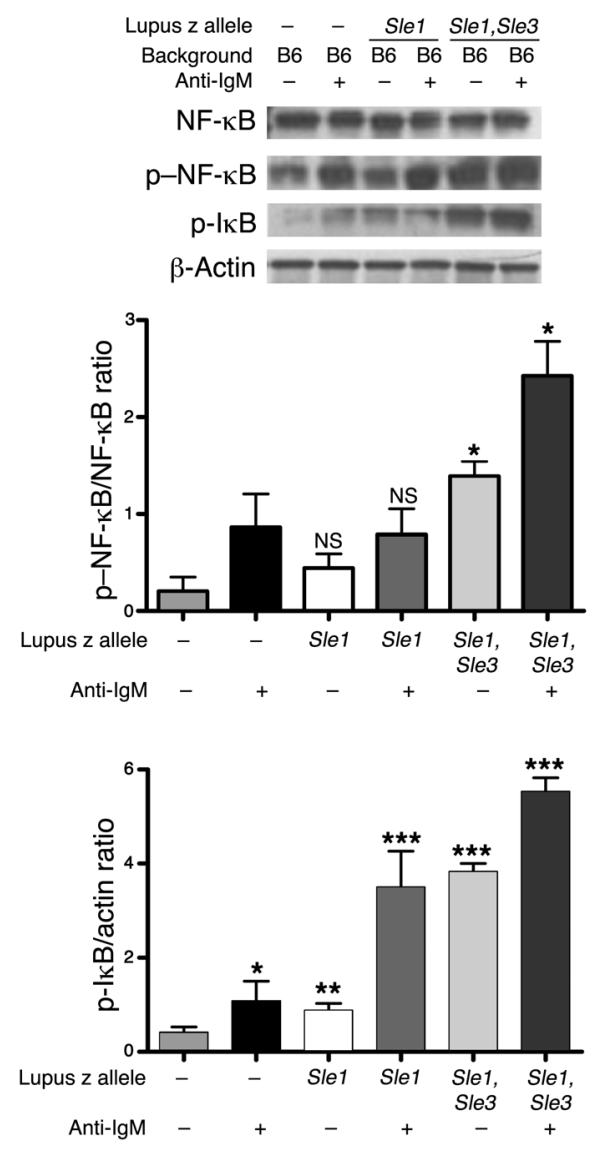

old B6.Sle ${ }^{\mathrm{z}}$.Sle $3^{\mathrm{z}} \mathrm{B}$ cells. In contrast, the levels of PKC $\alpha$ were progressively downregulated with age/disease in these mice (Table 1).

One may posit that the above signaling profiles that appear in unmanipulated lupus B cells, particularly with age, may represent the result of chronic $B$ cell activation by self antigens. To determine whether chronic BCR ligation of B6 B cells might recapitulate the signaling profile noted in lupus B cells, purified B6 splenic B cells were restimulated with anti-IgM following an earlier 48-hour round of prestimulation (in order to mimic chronic BCR stimulation). As can be noted from Figure 7A, "chronic" BCR ligation of normal B cells in vitro fails to recapitulate the signaling profiles noted in lupus B cells. Next, we considered the possibility that the signaling differences shown in Figures 1-6 may have occurred because the lupus B cells differed from B 6 B cells in terms of their subset composition. However, this did not appear to be the case since 2-monthold B6, B6.Sle $1^{\mathrm{z}}$, and B6.Sle $1^{\mathrm{z}}$.Sle ${ }^{\mathrm{z}}$ mice exhibited similar percentages of splenic follicular and mar-

Figure 4

$\mathrm{Bcl}-2$ family members in lupus B cells. Legend is as noted for Figure 1, except that various molecules in the Bcl-2 family were specifically examined. ${ }^{\star} P<0.05 ;{ }^{* *} P<0.01 ;{ }^{* \star *} P<0.001$.

\section{Figure 3}

Activation status of NF-кB pathway in lupus B cells. Legend is as noted for Figure 1, except that various molecules in the NF- $\mathrm{KB}$ axis were specifically examined. ${ }^{\star} P<0.05 ;{ }^{* \star} P<0.01$; ${ }^{\star * \star} P<0.001$.

ginal zone B cells (data not shown). To obtain a more definitive answer to this question, we flow-sorted small (i.e., excluding B cell

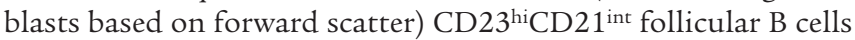
from the spleens of 2-month-old B6 and B6.Sle $1^{\mathrm{z}}$ mice and studied their signaling profiles.

As shown in Figure 7B, unmanipulated resting B6.Sle $1^{\mathrm{z}}$ follicular $B$ cells exhibited higher levels of $\mathrm{p}-\mathrm{AKT}$ and $\mathrm{p}$-Erk1/2 compared with the B6 controls. Importantly, these signaling molecules (particularly p-AKT) became more activated in B6.Sle $1^{\mathrm{z}}$ follicular B cells within 5 minutes of BCR cross-linking and remained elevated even 24 hours after stimulation compared with B6 follicular B cells. Collectively, these studies indicate that the hyperexpression of multiple signaling axes in lupus B cells may not simply be the consequence of chronic BCR ligation by autoantigens or differences in B cell subsets but may relate to intrinsic differences in BCR signaling thresholds (as examined in this study) or perhaps reflect the impact of other activated cells (such as T cell and myeloid cells) on these B cells in vivo.

To determine whether the signaling profiles exhibited by B6.Sle $1^{z}$ and B6.Sle ${ }^{\mathrm{z}}$.Sle ${ }^{\mathrm{z}} \mathrm{B}$ cells were representative of $\mathrm{B}$ cells from other mouse models of lupus, we determined the activation status of the implicated signaling axes in 2 other mouse models that develop spontaneous lupus: BXSB and MRL/lpr. These 2 strains were selected because they were genetically distinct from the B6.Sle $1^{z}$ and B6.Sle $1^{\mathrm{z}}$.Sle $3^{\mathrm{z}}$ strains and the related NZB/NZW and NZM2410 strains. Compared with cells from B6 mice, unmanipulated splenic $\mathrm{B}$ cells from BXSB males exhibited 3-fold more $\mathrm{p}$-AKT, 5-fold more p-mTOR, 3-fold more p-Bcl-2, 5-fold more p-Erk1/2, 2-fold more $\mathrm{p}-\mathrm{NF}-\kappa \mathrm{B}, 2$-fold more $\mathrm{p}-\mathrm{I \kappa} \mathrm{B} \alpha$, and 3-fold more p-STAT3 (Figure 8). Likewise, unmanipulated splenic B cells from MRL/lpr mice exhibited 3-fold more p-AKT, 8-fold more p-mTOR, 4-fold more p-Bcl-

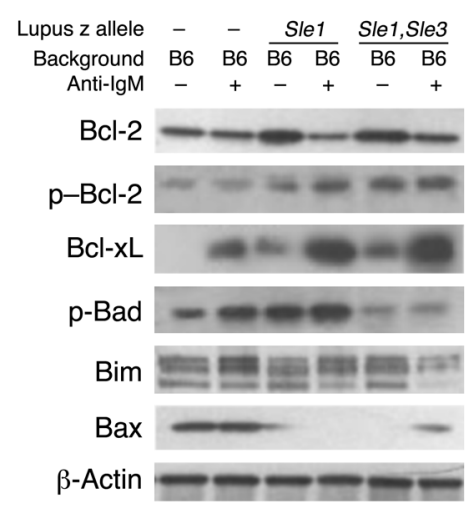


Table 1

Progressive activation of multiple signaling axes in lupus B cells

\begin{tabular}{|c|c|c|c|}
\hline Molecule & $\begin{array}{l}2 \text { months } \\
\text { B6.Sle1.Sle3z }\end{array}$ & $\begin{array}{c}4 \text { months } \\
\text { B6.Sle1.Sle3 }\end{array}$ & $\begin{array}{c}6 \text { months } \\
\text { B6.Sle1.Sle3 }\end{array}$ \\
\hline AKT & 198 & $200^{A}$ & $195^{A}$ \\
\hline p-AKT & 30 & $90^{B}$ & $151^{B}$ \\
\hline mTOR & 195 & $215^{A}$ & $207^{A}$ \\
\hline p-mTOR & 25 & $78^{B}$ & $95^{\mathrm{B}}$ \\
\hline Bcl-2 & 120 & $200^{c}$ & $202^{C}$ \\
\hline $\mathrm{p}-\mathrm{Bcl}-2$ & 50 & $102^{C}$ & $158^{\mathrm{C}}$ \\
\hline Erk1/2 & 285 & $280^{A}$ & $290^{A}$ \\
\hline p-Erk1/2 & 27 & $75^{\mathrm{B}}$ & $140^{\mathrm{B}}$ \\
\hline$N F-\kappa B$ & 200 & $195^{A}$ & $190^{\mathrm{A}}$ \\
\hline p-NF-кB & 70 & $155^{C}$ & $260^{B}$ \\
\hline$p-\left.\right|_{\kappa} B$ & 55 & $172^{D}$ & $283^{D}$ \\
\hline $\mathrm{PKC} \alpha$ & 150 & $45^{\mathrm{B}}$ & $50^{\mathrm{B}}$ \\
\hline CDK2 & 35 & $100^{B}$ & $211^{C}$ \\
\hline STAT3 & 150 & $130^{\mathrm{A}}$ & $165^{\mathrm{A}}$ \\
\hline p-STAT3 & 60 & $150^{c}$ & $185^{\mathrm{B}}$ \\
\hline
\end{tabular}

Anti-B220 bead-purified splenic B cells were isolated from 2-, 4-, and 6-month-old female B6.Sle1z.Sle3z mice, lysed, electrophoresed, and blotted using a panel of antibodies targeted to various molecules in the following axes: AKT/mTOR, Ras/MEK1/Erk1/2, NF-кB, Bcl-2, cell cycle, JAK/STAT, and PKC $\alpha$, some of which were specific for phosphorylated forms of the signaling molecules. The mean values of 3-6 individual samples (derived from independent mice) are shown. $P$ values shown pertain to a Student's $t$ test comparison of the older mice (4 months old or 6 months old) against the 2-month-old congenic samples. ${ }^{A} \mathrm{NS}$; ${ }^{\mathrm{B}} \mathrm{P}<0.01 ;{ }^{\mathrm{C}} P<0.05 ;{ }^{\mathrm{D}} P<0.001$

2, 8-fold more p-Erk1/2, 4-fold more p-NF-кB, 5-fold more p-IкB $\alpha$ and 5 -fold more p-STAT3 compared with the B6 controls (Figure $8)$. As noted in the B6.Sle congenic strains, PKC $\alpha$ was also reduced in the 2 additional lupus strains examined.

The observation that the same set of signaling axes was upregulated (or downmodulated) in multiple genetically distinct models of spontaneous lupus has several important implications. First, it provides strong molecular evidence that though lupus in different models (or individuals) may emanate from distinct genetic triggers, these different triggers may ultimately funnel through a shared series of signaling pathways that lead to disease. Second, these shared signaling axes represent attractive targets for therapeutic intervention. We proceeded to study the potential pathogenic role of the $\mathrm{AKT} / \mathrm{mTOR}$ axis in murine lupus because this axis was reproducibly upregulated in all examined lupus models and was activated early in disease. Moreover, a well-tolerated inhibitor of the mTOR kinase, RAD001, was already available. We decided to prophylactically treat 2-month-old B6.Sle ${ }^{z}$.Sle $3^{z}$ lupus mice with RAD001. At this age, these mice are disease-free (ref. 21 and T. Wu

\section{Figure 5}

Cell-cycle proteins and other signaling axes in lupus $B$ cells. Legend is as noted for Figure 1, except that cell cycle proteins were specifically examined. ${ }^{\star} P<0.05 ;{ }^{* *} P<0.01 ;{ }^{* *} P<0.001$. and C. Mohan, unpublished observations). Given the observation that this axis undergoes profound upregulation from 2 months of age (Table 1), we decided to treat these mice with RAD001 over a 2 -month period from the age of 2 months to the age of 4 months, at which point the mice were sacrificed and phenotyped.

B6.Sle $1^{\mathrm{z}}$.Sle ${ }^{\mathrm{z}}$ mice treated with RAD001 had significant decreases in spleen size and cellularity, with $40-60 \%$ reduction in the absolute number of $\mathrm{B}$ cells and activated $\mathrm{T}$ cells compared with that in placebo-treated mice (Figure 9). In addition, RAD001-treated mice exhibited significant reduction in serum autoantibody levels, including levels of anti-dsDNA and anti-glomerular antibodies (Figure 9). Finally, the treated mice developed significantly less renal disease, as demonstrated by reduced proteinuria, blood urea nitrogen and glomerulonephritis (Figure 9). As anticipated, the RAD001-treated mice, when compared with placebo-treated controls, exhibited significant reduction in the levels of p-mTOR (reduced 2-fold) and a key downstream target, p-P70S6K (reduced 2-fold) (Figure 10). Surprisingly, the RAD001-treated mice also exhibited a pronounced dampening of p-AKT as well as several other signaling pathways as exemplified by a 2 -fold reduction in $\mathrm{p}$-Erk $1 / 2$, a 2 -fold reduction in $\mathrm{p}-\mathrm{NF}-\kappa \mathrm{B}$, a 3 -fold reduction in $\mathrm{p}-\mathrm{Bcl}-2$, a 2 -fold reduction in $\mathrm{CDK} 2$, and a 2 -fold reduction in p-STAT3 (Figure 10). Hence, targeting the AKT/mTOR axis has the capacity to dampen several additional signaling cascades that are also upregulated in murine lupus B cells.

\section{Discussion}

The present report offers what we believe is the first comprehensive view of multiple signaling axes in B cells as lupus evolves. Interestingly, multiple signaling pathways are observed to fire up in B cells, correlating well with disease progression. Mice with more severe disease, either as a consequence of increased genetic load (e.g., B6.Sle ${ }^{\text {z. Sle }} 3^{z}$ versus B6.Sle $1^{z}$ ) or as a function of age, clearly exhibited a quantitative change in the dysregulation of several signaling axes in their splenic B cells. The observation that a remarkably similar signaling profile was observed in multiple genetically distinct murine lupus strains indicates that these shared networks may be
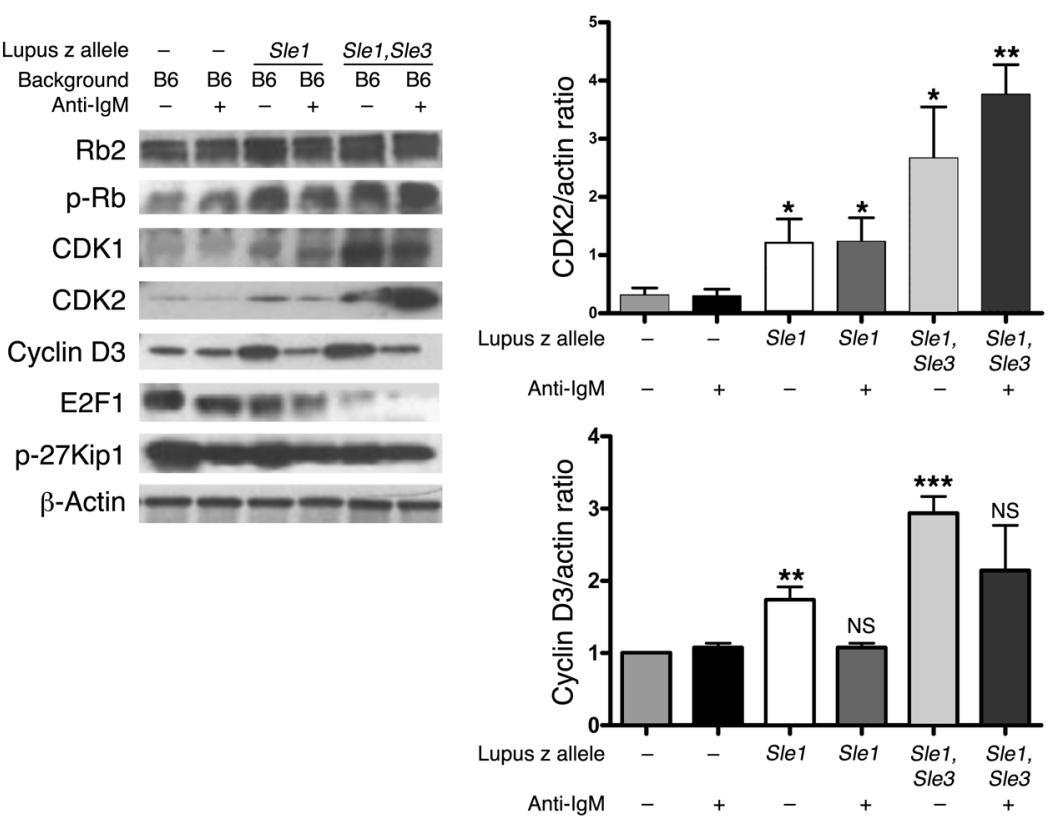


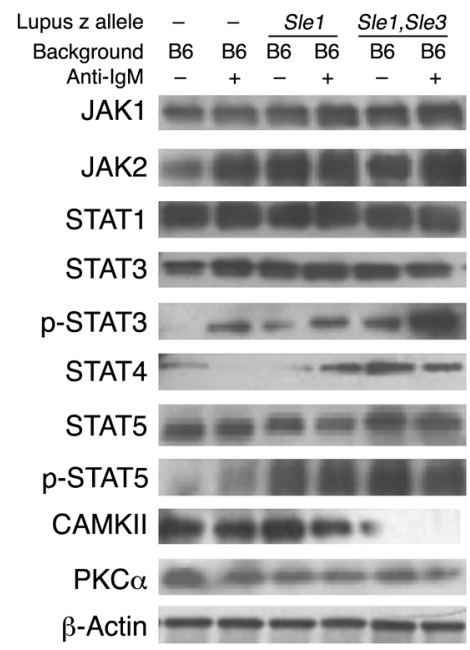

playing critical roles in disease pathogenesis in multiple genetic backgrounds independent of the initiating genetic triggers.

Several reports using engineered mouse models have established a central role for the PI3K/AKT/mTOR axis in the differentiation of peripheral B cell subsets and $\mathrm{T}$ cell homeostasis $(7,29)$. Upregulating the PI3K/AKT/mTOR pathway either by overexpression of PI3K in T cells (7) or by induction of PTEN haploinsufficiency (7) results in the development of similar lymphoproliferative and autoimmune disorders. On the other hand, deletion of the $\mathrm{I}_{\mathrm{B}}$-PI3K $\gamma$ isoform decreases survival of pathogenic $\mathrm{CD}^{+}$memory cells, selectively inhibiting lupus development (30). In line with the above studies, the level of phosphorylation of AKT in unstimulated B cells was significantly elevated in B6.Sle ${ }^{\mathrm{z}}$, B6.Sle $1^{z} . S l e 3^{z}, \mathrm{MRL} /$ lpr, and BXSB lupus mice, even at the age of 2 months, prior to disease, and became progressively more activated in step with the disease. AKT is a serine/threonine kinase that regulates cell growth, survival, metabolism, and cell-cycle progression (31). Activated AKT is phosphorylated at 2 sites: PI3K-dependent kinase-1 (PDK1) phosphorylates T308 in the catalytic domain whereas mTOR complex 2 (mTORC2) phosphorylates S473 in the hydrophobic site (32). In our experiments, phosphorylation at the T308 site was more pronounced, suggesting an upregulation of the PDK1-mediated pathway.

Among the MAPKs, we found identical patterns of upregulation of 2 different kinases, Erk1/2 and p38, but not JNK. Significant activation of these kinases was noted in anti-IgM-stimulated B cells of both congenic strains and was constitutively activated in B6.Sle $1^{\mathrm{z}}$.Sle $3^{\mathrm{z}} \mathrm{B}$ cells. As far as the Sle 1 genetic interval is concerned, the upregulation of Erk $1 / 2$ is likely to be the consequence of (yet to be defined) genes within the Sle1 $a$ and/or Sle1b intervals, based on a recent report that B6.Sle1 $\mathrm{b}^{z} \mathrm{~B}$ cells expressed elevated Erk2 (33). To our knowledge, these constitute the first

\section{Figure 7}

A

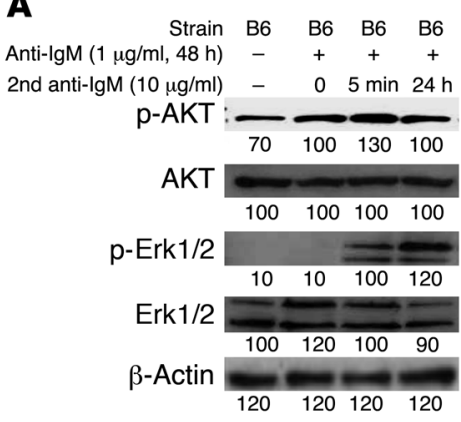

B

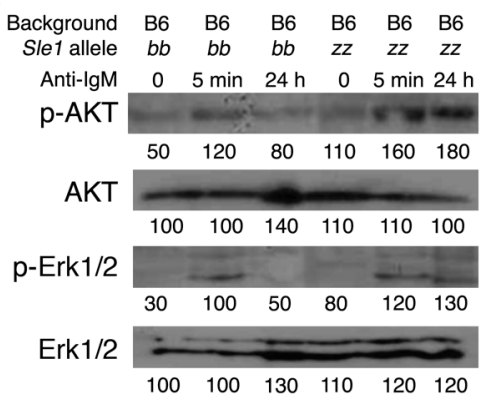

Activation status of AKT and Erk1/2 in total splenic B cells and resting follicular $B$ cells. Anti-B220 bead-purified splenic B cells $(\mathbf{A})$ and purified follicular splenic $B$ cells obtained by flow cytometry-based cell-sorting $(B)$ were isolated from 2-month-old female B6 and B6.Sle $1 z$ mice. Following an initial stimulation with a lower concentration of anti-lgM $F\left(a^{\prime}\right)_{2}(1 \mu \mathrm{g} / \mathrm{ml})$ for 48 hours, cells were either immediately lysed or were reexposed to a higher concentration of anti-lgM $F\left(a^{\prime}\right)_{2}$ $(10 \mu \mathrm{g} / \mathrm{ml})$ for 5 minutes or 24 hours and then lysed. Lysates were electrophoresed and blotted using antibodies against the total or phosphorylated forms of AKT and Erk $1 / 2$. The mean band intensity values as analyzed by ImageJ are indicated below each band. 


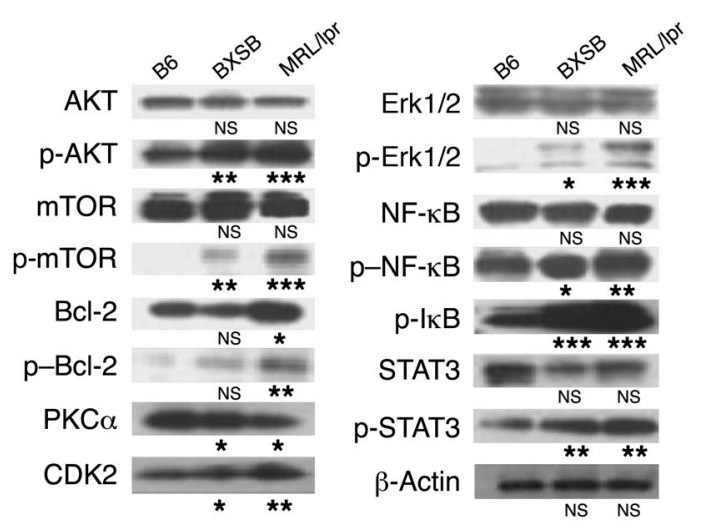

STAT proteins are transcription factors that provide a direct link between the cytokine receptors and cytokine-induced gene transcription. There is very limited information in the literature concerning the role of various STAT molecules in lupus pathogenesis. STAT4 and STAT6 transcription factors play a role in determining the Th1/Th2 cytokine balance. A recent report showed that STAT4-deficient NZM2328 mice developed accelerated nephritis and increased mortality in the absence of high levels of autoantibodies including anti-dsDNA antibodies (39). In contrast, STAT6deficient NZM2328 mice displayed a significant reduction in the incidence of kidney disease, with increased survival, in spite of the presence of high levels of anti-dsDNA antibodies (39). In our study, although STAT4 and STAT6 were not elevated (data not shown), elevated phosphorylated STAT3 was seen in B cells of bicongenic B6.Sle ${ }^{z}$.Sle $3^{z}$ mice whereas constitutively upregulated STAT5 was seen in B cells of both congenic strains. In the related B6.Sle1abz congenic strain, phosphorylated STAT3 has been reported to be elevated as a result of induction by IL-6 (33). Further studies are warranted to examine the pathogenic role of these different STAT molecules in B cells and non-B cells in the B6.Sle congenic models.

Limited information is available on the role of the NF-кB/ІкB kinase axis in lupus. We believe this is the first report examining NF- $\mathrm{KB}$ status in lupus B cells. Surprisingly, however, compared with that in normal cells, the activation of NF- $\kappa \mathrm{B}$ in freshly isolated $T$ cells from SLE patients was significantly decreased (40).

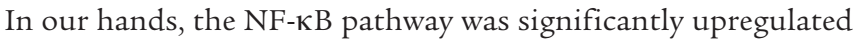
in B cells from unmanipulated and anti-IgM-stimulated bicongenic mice as well as in BXSB and MRL/lpr mice but not in B6.Sle1 ${ }^{z}$ monocongenic mice. Since NF-KB was upregulated only in the strains that develop severe lupus (but not the B6.Sle1z congenic strain), this lends support to the notion that NF-кB activation (for example, through innate triggers channeled via various TLRs) may be necessary for severe lupus to ensue.

In contrast with the above pathways, considerable information is available concerning the roles of Bcl-2 family members in lupus. In engineered murine lupus models, the enforced expression of the antiapoptotic molecule Bcl-2 is associated with extended B cell survival, follicular lymphoproliferation, and development of autoimmune disease $(8,41,42)$. In humans, $\mathrm{Bcl}-2$ is elevated in both $\mathrm{B}$ and $\mathrm{T}$ cells from SLE patients, resulting in faulty apoptosis (26). In addition, a positive correlation between serum Bcl-2 levels and lupus activity has been reported (43). Our present findings extend these observations by demonstrating that murine lupus is associated with the upregulation of the antiapoptotic molecule Bcl-2 with a concomitant decrease of proapoptotic molecules, such as

\section{Figure 8}

Status of selected signaling pathways in other lupus models. AntiB220 bead-purified splenic $B$ cells were isolated from female B6, male $\mathrm{BXSB}$, and female MRL/lpr mice, aged 2-3 months, lysed (without deliberate BCR cross-linking), electrophoresed, and blotted using a panel of antibodies targeted to the indicated signaling molecules, some of which were specific for the phosphorylated forms of the signaling molecule, as noted. The $P$ values shown pertain to a Student's $t$ test comparison of the BXSB or MRL/Ipr values with the B6 value. ${ }^{\star} P<0.05 ;{ }^{\star \star} P<0.01 ;{ }^{\star \star \star} P<0.001$.

Bim, Bax, and Bad. At least some of these alterations may be occurring as a consequence of upregulation of various upstream signaling axes. For instance, AKT, which is upregulated in lupus B cells, is known to control cell survival by inactivating $\operatorname{Bad}(44,45)$.

Perhaps not surprisingly, the upregulation of the different signaling axes described above was also associated with significant changes in several downstream molecules necessary for cell-cycle progression. Members of the Cip/Kip family, including p $21^{\text {CIP-1, }}$

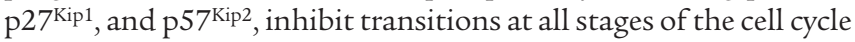
by binding to the entire cyclin/CDK holoenzyme (46). An important target of activated cyclin/CDK complexes is Rb. Hypophosphorylated Rb blocks E2F, which is necessary for transcription of genes required for $\mathrm{S}$-phase progression. Though we have not executed an exhaustive screen of all Cip/Kip and cyclin/CDK members, our findings suggest reduced Cip/Kip leads to activation of $\mathrm{CDK} /$ cyclin and hyperphosphorylation of $\mathrm{Rb}$, which leads in turn to reduced E2F, may constitute an important downstream axis in lupus B cells, with this cascade of events becoming progressively more accentuated with disease. We believe this is the first report on the status of this axis in lupus B cells, either in mice or in humans. It appears that this axis may also be suitable for therapeutic intervention. Of particular note, Goulvestre et al. have reported that the administration of a peptidyl mimic of $\mathrm{p} 21^{\mathrm{WAF} 1 / \mathrm{CIP} 1}$ inhibited the progression of lupus-like syndrome in $(\mathrm{NZB} \times \mathrm{NZW}) \mathrm{F}_{1}$ mice $(47)$.

Understanding the complexity of and possible interactions among these signaling pathways is critical in deciding where (i.e., at which signaling node) to intervene therapeutically. In this study, we focused on targeting the PI3K/AKT/mTOR axis for a variety of reasons. The PI3K/AKT/mTOR axis has been extensively studied in cancer research because a substantial fraction of human tumors have mutations or epigenetic aberrations in the components of this pathway (48-50). Multiple inhibitors targeting different components of the $\mathrm{PI} 3 \mathrm{~K} / \mathrm{AKT} / \mathrm{mTOR}$ pathway are being generated, but the most developed among these are the mTOR inhibitors. The highly conserved serine/threonine kinase mTOR is a key regulatory kinase involved in protein synthesis control, growth, cell-cycle progression, and angiogenesis $(49,51,52)$. Recent findings indicate that the PI3K and Ras/MEK1/Erk1/2 signaling pathways converge to activate mTOR and subsequently promote cell growth (33), suggesting that mTOR represents a critical growth-control node. Rapamycin and its derivatives RAD001, CCI-779 (temsirolimus), and AP-23573 have shown activity against various types of cancers in phase I and II trials (53-55). Rapamycin and its derivatives form a complex with FKBP12, a 11.8-kDa immunophilin; however, the exact mechanism of inhibition of mTORC1 by FKBP12-rapamycin 

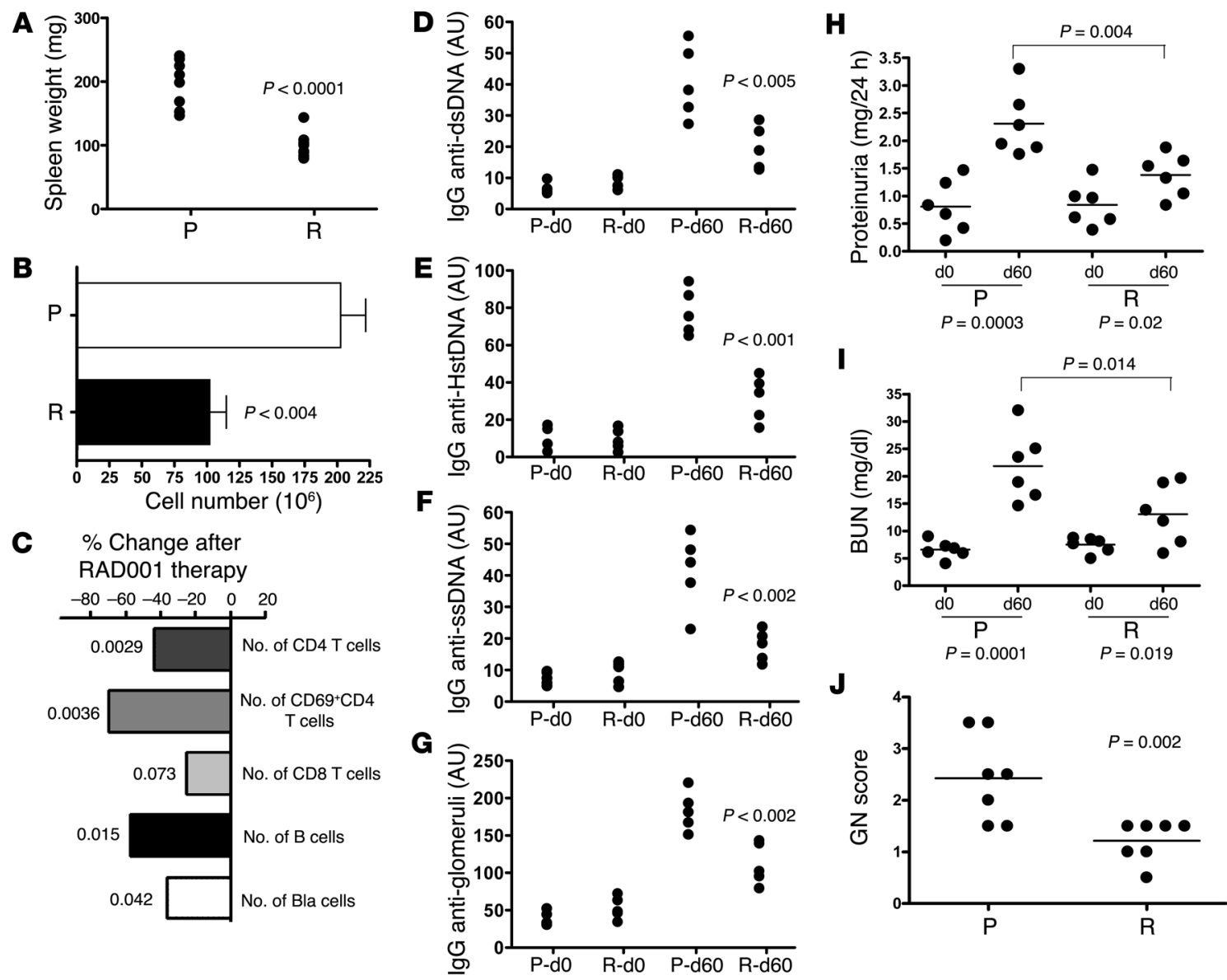

Figure 9

Impact of mTOR blockade on B6.Sle 1z.Sle3z lupus. Shown are changes in spleen weight (A), total splenocyte counts (B), percentage change in the numbers of different splenic cell subsets $(\mathbf{C})$, anti-nuclear autoantibodies as determined by ELISA (D-G), proteinuria (H), blood urea nitrogen (BUN) (I), and kidney pathology (J). GN, glomerulonephritis; P, placebo; R, RAD001; d, days after treatment. $P$ values shown pertain to a Student's $t$ test comparison of the RAD001-treated congenic samples with the placebo-treated congenic samples.

complex remains unclear. It appears that this inhibition is executed by multiple mechanisms, including inhibition of mTORC1 autophosphorylation (56) and dissociation of raptor-mTOR (57). Thus, FKBP12-rapamycin might be inhibiting the intrinsic kinase activity of mTOR and denying it access to its substrates.

In our present study, we find RAD001 to be very effective at ameliorating all aspects of the disease in lupus-prone mice, including autoantibodies, end-organ pathology, and cellular phenotypes. Somewhat striking was the observation that RAD001-treated mice exhibited dampening of multiple signaling axes besides mTOR. Perhaps this should not be a surprise, given that several upstream axes do funnel through mTOR (31) and that mTOR activity can regulate several downstream targets, either directly or indirectly. Importantly, others have shown that inhibition of mTORC1 by rapamycin and RAD001 blocks the cell cycle in the late G1/S phase by downregulating D1 cyclin/ CDK complexes (58). Furthermore, RAD001 was reported to prevent activation of NF- $\kappa \mathrm{B}$ by stabilizing its major inhibitor, $\mathrm{I} \kappa \mathrm{B} \alpha(59)$. Hence, crosstalk between different signaling pathways impinging upon mTOR and possibly the multiple sites of action of RAD001 might help explain the dampening effect of this drug on multiple signaling pathways.
Finally, an important issue that warrants study is how the genes and phenotypes associated with Sle $1^{z}$ and Sle $3^{z}$ may relate to the observed signaling changes. We have recently reported that Sle $1 b^{z}$ (which represents the strongest sublocus within Sle $1^{z}$ ) impedes deletion and receptor editing of immature B cells due to a polymorphism in the Ly108 gene (60). This may result in the persistence of antinuclear B cells in the primary repertoire, which are then likely to be incessantly stimulated by self-nuclear antigens. Signaling via polymorphic variants of Ly108 in immature B cells and continuing stimulation of self-reactive mature B cells in the periphery may together contribute to the elevated levels of PI3K/AKT/ mTOR and other signaling axes in these mice (K.R. Kumar et al., unpublished observations). More importantly, the short-term stimulation studies using resting follicular B cells (Figure 7B) suggest that lupus susceptibility genes (such as Ly108) may modulate the BCR signaling threshold so that these B cells intrinsically respond differently to BCR cross-linking.

The second genetic component examined in this work, Sle $3^{z}$, contributes to lupus through the generation of hyperactive dendritic cells (24). We now know that incessant TLR-mediated triggering of Sle $3^{z}$-bearing dendritic cells may be responsible for the elevated PI3K/AKT/mTOR and NF-кB axes noted in these cells 


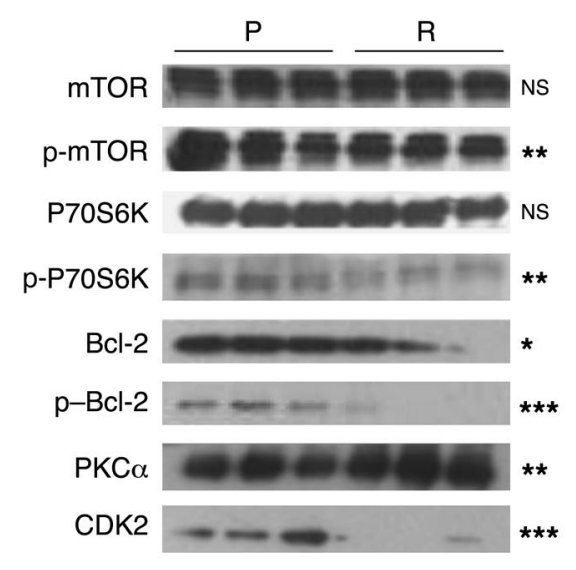

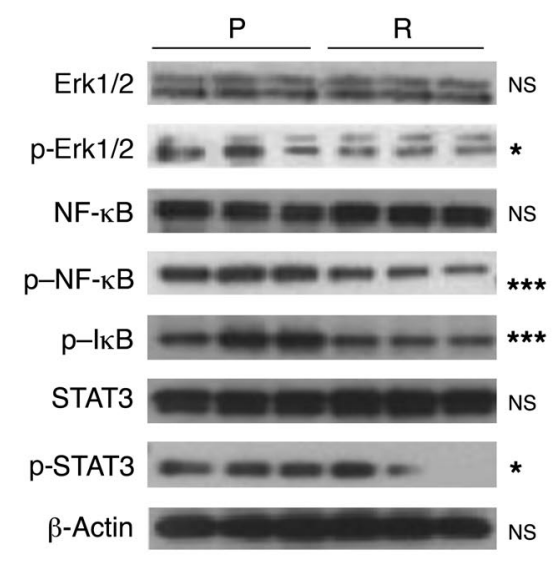

\section{Figure 10}

mTOR blockade dampens multiple signaling axes in lupus. Anti-B220 bead-purified splenic $B$ cells were isolated from 4-month-old female B6.Sle $1^{z}$.Sle ${ }^{z}$ mice (treated with RAD001 or placebo), lysed, electrophoresed, and blotted using a panel of antibodies targeted to the indicated signaling molecules, some of which were specific for the phosphorylated forms of the signaling molecules, as noted. $P$ values shown pertain to a Student's $t$ test comparison of the RAD001-treated congenic samples with the placebo-treated congenic samples. ${ }^{*} P<0.05$; ${ }^{* *} P<0.01 ;{ }^{* *} P<0.001$.

(J. Zhu et al., unpublished observations). Interestingly, rapamycin has been shown to partially inhibit many functions of dendritic cells by inhibiting mTOR $(61,62)$. Since dendritic cells play a crucial role in the perpetuation of autoimmunity, both systemically and locally, it is likely that the observed beneficial effect of RAD001 in the congenic lupus strain was in part due to the inhibition of Sle $3^{z}$-bearing dendritic cells in these mice. Importantly, the upregulation of the same signaling axes (including PI3K/AKT/ mTOR, NF- $\mathrm{B}$, and MAPK) in several genetically diverse mouse strains with severe lupus suggest that these molecular profiles may be generalized features of disease progression in lupus. The observation that genetically diverse triggers can give rise to downstream signaling profiles that are apparently universal in lupus raises hope from the perspective of therapy.

\section{Methods}

Mice. C57BL/6 (B6), MRL/lpr, and BXSB mice were obtained from The Jackson Laboratory and were then bred in our animal colony. The derivation of B6 congenic mice bearing different NZM2410-derived lupus susceptibility intervals has been detailed previously (63). B6.Sle $1^{\mathrm{z}}$ mice are C57BL/6 mice congenic for a 37-cM interval on murine chromosome 1 , spanning the $95 \%$ confidence interval flanking the $\mathrm{z}$ allele of Sle 1 , with termini at D1MIT101 and D1MIT55. B6.Sle $3^{z}$ mice are C57BL/6 mice congenic for a $40-\mathrm{cM}$ interval on murine chromosome 7 , spanning the $95 \%$ confidence interval flanking the $\mathrm{z}$ allele of Sle3, with termini at D7MIT56 and D7MIT62. Both congenic intervals are derived from the NZW parent of the NZM2410 strain. B6.Sle $1^{z} \cdot$ Sle $^{z}$ mice, bicongenic for both the study intervals, were derived by intercrossing the monocongenic strains and breeding and selecting offspring that were homozygous across both susceptibility intervals. All mice used for this study were bred and housed in a specific pathogen-free colony at University of Texas Southwestern Medical Center Department of Animal Resources. Both male and female mice were used, and any observed sex differences are noted. These experiments have been approved by the Institutional Animal Care and Use Committee (IACUC) at the University of Texas Southwestern Medical Center.
Flow cytometric analysis and antibodies. Splenocytes were depleted of red blood cells using lysis buffer (containing $0.15 \mathrm{M} \mathrm{NH}_{4} \mathrm{Cl}, 10 \mathrm{mM} \mathrm{KHCO}_{3}, 0.1$ $\mathrm{mM} \mathrm{Na}{ }_{2}$ EDTA, pH 7.2), and single-cell suspensions were prepared for flow cytometric analysis. $\mathrm{LN}$ cells were obtained from the inguinal sites and crushed to obtain single-cell suspensions. Peritoneal cavity cells were obtained by flushing the peritoneal cavities with fresh medium. Flow cytometric analysis was performed as described previously (22). The mean linear units on the forward-scatter channel were used as indicators of cell size. $\mathrm{CD}^{+} \mathrm{T}$ cells that did not express CD4 or CD8 were classified as double-negative T cells. $\mathrm{B} 220^{+}$cells were further subdivided into B1a and B2 cells based on whether or not they expressed surface CD5. In addition, the percentages of follicular B cells $\left(\mathrm{B} 220^{+} \mathrm{CD} 23^{\text {hi }} \mathrm{CD} 21^{\mathrm{int}}\right)$ and marginal zone B cells $\left(\mathrm{B} 220^{+} \mathrm{CD} 23^{1 \circ} \mathrm{CD} 21^{\mathrm{hi}}\right)$ were defined. $\mathrm{B} 220^{+} \mathrm{CD} 21^{\text {lo }} \mathrm{CD} 23^{\text {lo }} \mathrm{B}$ cells were identified as being preplasmablasts predominantly, based on their surface expression of syndecan-1, CD43, and other markers as indicated.

Purification of splenic follicular B cells. Spleens were harvested from euthanized mice using institution-approved protocols. Single-cell suspension was prepared by crushing spleens between frosted glass slides. rbc were lysed using ACK Lysing Buffer (Invitrogen) followed by 2 washes in PBS plus 0.5\% BSA. Naive B cells were purified by negative selection from this total splenocyte suspension with CD43 microbeads (Miltenyi Biotec) using the manufacturer-recommended protocol. In some studies, these cells were further labeled with pretitrated dilutions of fluorochrome-labeled antibodies against CD21 and CD23 and subjected to flow cytometry-based cell sorting (FACSAria; $\mathrm{BD})$ to obtain follicular B cells (B220+CD21 $1^{\text {int }} \mathrm{CD} 23^{\text {hi }}$ ).

ELISA for autoantibodies. The anti-dsDNA, anti-histone, and antihistone/DNA ELISA assays were carried out as previously described (22). Raw OD was converted to $\mathrm{U} / \mathrm{ml}$ using a positive control serum derived from a B6.Sle1.lpr mouse; reactivity of a 1:100 dilution of this serum was arbitrarily set to $100 \mathrm{U} / \mathrm{ml}$. Test sera with reactivity stronger than the standard were diluted further and reassayed. Glomerular-binding ELISA was performed as described previously (22), using sonicated rat glomeruli as substrate.

Commercial immunoblot screening. Splenocytes isolated from gendermatched 6-month-old B6.Sle1 ${ }^{z}$, B6.Sle1 ${ }^{z}$.Sle ${ }^{z}$ and B6 controls were scanned for the expression of several different signaling molecules, using KPKS-1.1 and KPSS-4.1 multiplexed Western blot assays by Kinexus. BD PowerBlot scanning was also performed using a customized panel of antibodies (Becton Dickinson), beginning with similar cells. Subsequently, these assays were carried out in-house.

Western blot analysis. Splenic B cells were isolated by using anti-B220 magnetic beads (Miltenyi Biotec); the resulting B cells were more than $95 \%$ pure. For the stimulation studies, B cells were stimulated with AffiniPure $\mathrm{F}\left(\mathrm{ab}^{\prime}\right)_{2}$ goat anti-mouse IgM $(10 \mu \mathrm{g} / \mathrm{ml}$; Jackson ImmunoResearch Laboratories Inc.) for 24 hours. B cells were then lysed using $20 \mathrm{mM}$ Tris- $\mathrm{HCl}$ (pH 7.5), $150 \mathrm{mM} \mathrm{NaCl}, 1 \mathrm{mM} \mathrm{Na}{ }_{2}$ EDTA, $1 \mu \mathrm{g} / \mathrm{ml}$ leupetin, $1 \%$ Triton X-100, $1 \mathrm{mM}$ PMSF, and $1 \mathrm{mM} \mathrm{Na}_{3} \mathrm{VO}_{4}$. Total protein was quantified by the Bradford assay, and $10 \mu \mathrm{g}$ was loaded per lane onto SDS-PAGE gels. The following primary antibodies were obtained from Cell Signaling Technology (name, product no.): CaMKII, 3362; E2F-1, 3742; p27 ${ }^{\mathrm{Kip} 1}$, 2552; p-Rb ${ }^{\mathrm{S} 807 / 811}$, 9308; p-Stat3 ${ }^{\mathrm{S} 727}$, 9134; 


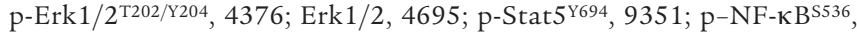
3031; p-IкB ${ }^{\mathrm{S} 32 / 36}, 9246$; p-MEK1/2 ${ }^{\mathrm{S} 217 / 221}$, 9121; MEK1/2, 9122; p-P38/ MAPK $^{\mathrm{T} 180 / Y 182}$, 9216; mTOR, 2972; p-mTOR ${ }^{\mathrm{S} 2448}$, 2971; 4E-BP1, 9452; p-4E-BP1 ${ }^{\mathrm{T} 37 / 46}, 9459$; P70S6 kinase, 9202; p-p70S6 kinase ${ }^{\mathrm{T} 389}, 9205$; AKT, 9272; p-AKT ${ }^{\mathrm{T} 308}$, 9275; Bax, 2772; Bcl-xL, 2762; and p-Jak2 ${ }^{\mathrm{Y} 221}$, 3774. The following primary antibodies were purchased from BD Biosciences - Pharmingen (name, product number): JAK1, 610231; CDK2, 610145; Rb2, 610261; cyclin D3, 610279; PKC $\alpha$, 610107; Stat1, 610185; Stat3, 610189; Stat4, 610926; and Stat5, 610191. Antibodies against $\mathrm{p}-\mathrm{AKT}^{\mathrm{S} 473}$ (44-622, Biosource International; Invitrogen), $\beta$-actin (RGM2, Advanced ImmunoChemical Inc.), Bcl-2 (sc-23960, Santa Cruz Biotechnology Inc.), p-Bcl-2 ${ }^{\mathrm{T} 129}$ (29658, AnaSpec), and Bim/Bad (AAP-330, Stressgen Biotechnologies) were also used. HRP-conjugated secondary antibodies and the ECL-Plus Detection Kit (GE Healthcare) were used to develop blots. Respective band intensities were measured using ImageJ (version 1.37; http://rsb.info.nih.gov/ij), and normalized against the corresponding $\beta$-actin levels. Where samples from different strains were compared, normalized band intensities were expressed as ratios, relative to the corresponding $\mathrm{B} 6$ levels.

In vivo treatment. RAD001 ( $2 \% \mathrm{w} / \mathrm{w}$ stock solution; Novartis) was diluted in PBS to $1 \mathrm{mg} / \mathrm{ml}$. Two-month-old mice were administered a final dose of $10 \mathrm{mg} / \mathrm{kg}$ RAD001 or vehicle alone (i.e., placebo), by oral gavage $4 \times /$ week for a period of 2 months. Serum and 24 -hour urine samples were obtained on days 0,14 , and 60 . All serum samples were assayed for autoantibodies by ELISA, and urine samples were assayed for total protein as described above. On day 60, upon sacrifice, the cellular composition of the spleen and nodes was determined by flow cytometry, and the kidneys were examined for pathology, as described above. In addition, the expression of various signaling molecules in the spleens of the treated mice was assayed by Western blot, as described above.
Renal disease. Mice were monitored at 3 months and 6 months of age for evidence of nephritis. The 24-hour urine samples were collected using metabolic cages. The total amount of urinary protein was assayed using a Coomassie-based assay (Pierce Biotechnology). Upon sacrifice, kidneys were fixed, sectioned, and stained with H\&E and PAS. At least 100 glomeruli were examined per section by light microscopy for evidence of inflammation and/ or tissue damage and graded as previously described (64) in a blinded fashion. The occurrence of any mesangiopathic, capillary hyaline, proliferative, membranous, or crescentic glomerular changes was also noted.

Statistics. Statistical comparisons were performed using the paired or unpaired Student's $t$ test, as appropriate, using SigmaStat (Jandel scientific). For all experiments, mean/SEM is also depicted. A $P$ value less than 0.05 was considered significant.

\section{Acknowledgments}

This work is supported in part by NIH grants R01 AR44894 and R01 AR50812 as well as the Alliance for Lupus Research. A.B. Satterthwaite is a Southwestern Medical Foundation scholar in biomedical research. We would like to acknowledge Madhavi Bhaskarabhatla for technical help and Novartis for helpful discussions and for providing RAD001.

Received for publication September 20, 2006, and accepted in revised form May 8, 2007.

Address correspondence to: Chandra Mohan, Department of Internal Medicine/Rheumatology, UT Southwestern Medical Center, Mail Code 8884, Y8.204, 5323 Harry Hines Boulevard, Dallas, Texas 75390-8884, USA. Phone: (214) 648-9675; Fax: (214) 6487995; E-mail: chandra.mohan@utsouthwestern.edu.
1. Kotzin, B.L. 1996. Systemic lupus erythematosus. Cell. 85:303-306.

2. Lipsky, P.E. 2001. Systemic lupus erythematosus: an autoimmune disease of B cell hyperactivity. Nat. Immunol. 2:764-766.

3. Mohan, C., Morel, L., Yang, P., and Wakeland, E.K. 1997. Genetic dissection of systemic lupus erythematosus pathogenesis: Sle2 on murine chromosome 4 leads to B cell hyperactivity. J. Immunol. 159:454-465.

4. Klinman, D.M. 1990. Polyclonal B cell activation in lupus-prone mice precedes and predicts the development of autoimmune disease. J. Clin. Invest. 86:1249-1254

5. Haraldsson, M.K., et al. 2005. Autoimmune alterations induced by the New Zealand Black Lbw2 locus in BWF1 mice. J. Immunol. 174:5065-5073.

6. Nijnik, A., et al. 2006. Spontaneous B cell hyperactivity in autoimmune-prone MRL mice. Int. Immunol. 18:1127-1137.

7. Borlado, L.R., et al. 2000. Increased phosphoinositide 3-kinase activity induces a lymphoproliferative disorder and contributes to tumor generation in vivo. FASEB J. 14:895-903.

8. Strasser, A., et al. 1991. Enforced BCL2 expression in B-lymphoid cells prolongs antibody responses and elicits autoimmune disease. Proc. Natl. Acad. Sci. U. S. A. 88:8661-8665.

9. Di Cristofano, A.P., et al. 1999. Impaired Fas response and autoimmunity in Pten $+/-$ mice. Science. 285:2122-2125.

10. Theofilopoulos, A.N., and Dixon, F.J. 1985. Murine models of systemic lupus erythematosus. $A d v$. Immunol. 37:269-390.

11. Cohen, P.L., and Eisenberg, R.A. 1991. Lpr and gld: single gene models of systemic autoimmunity and lymphoproliferative disease. Annu. Rev. Immunol. 9:243-269.
12. Rudofsky, U.H., Evans, B.D., Balaban, S.L., Mottironi, V.D., and Gabrielesen, A.E. 1993. Differences in expression of lupus nephritis in New Zealand mixed $\mathrm{H}-2 \mathrm{z}$ homozygous inbred strains of mice derived from New Zealand black and New Zealand white mice. Origins and initial characterization. Lab. Invest. 68:419-426.

13. Sobel, E.S., Satoh, M., Chen, Y., Wakeland, E.K., and Morel, L. 2002. The major murine systemic lupus erythematosus susceptibility locus Sle1 results in abnormal functions of both B and T Cells. J. Immunol. 169:2694-2700.

14. Sobel, E.S., Mohan, C., Morel, L., Schiffenbauer, J., and Wakeland, E.K. 1999. Genetic dissection of SLE pathogenesis: adoptive transfer of Sle 1 mediates the loss of tolerance by bone marrow-derived B cells. J. Immunol. 162:2415-2421.

15. Merino, R., Fossati, L., Lacour, M., and Izui, S. 1991. Selective autoantibody production by Yaa + B cells in autoimmune Yaa(+)-Yaa- bone marrow chimeric mice. J. Exp. Med. 174:1023-1029.

16. Morel, L., Blenman, K.R., Croker, B.P., and Wakeland, E.K. 2001. The major murine systemic lupus erythematosus susceptibility locus, Sle1, is a cluster of functionally related genes. Proc. Natl. Acad. Sci. U. S. A. 98:1787-1792.

17. Wakeland, E.K., Liu, K., Graham, R.R., and Behrens, T.W. 2001. Delineating the genetic basis of systemic lupus erythematosus. Immunity. 15:397-408.

18. Kono, D.H., and Theofilopoulos, A.N. 2000. Genetics of systemic autoimmunity in mouse models of lupus. Int. Rev. Immunol. 19:367-387.

19. Morel, L., et al. 2000. Genetic reconstitution of systemic lupus erythematosus immunopathology with polycongenic murine strains. Proc. Natl. Acad. Sci.U.S. A. 97:6670-6675.

20. Mohan, C., Alas, E., Morel, L., Yang, P., and Wakeland, E.K. 1998. Genetic dissection of SLE patho- genesis. Sle 1 on murine chromosome 1 leads to selective loss of tolerance to $\mathrm{H} 2 \mathrm{~A} / \mathrm{H} 2 \mathrm{~B} / \mathrm{DNA}$ subnucleosomes. J. Clin. Invest. 101:1362-1372.

21. Mohan, C., et al. 1999. Genetic dissection of lupus pathogenesis: a recipe for nephrophilic autoantibodies. J. Clin. Invest. 103:1685-1695.

22. Shi, X., Xie, C., Kreska, D., Richardson, J.A., and Mohan, C. 2002. Genetic dissection of SLE: Sle1 and FAS impact alternate pathways leading to lymphoproliferative autoimmunity. J. Exp. Med. 196:281-292.

23. Sobel, E.S., Mohan, C., Morel, L., Schiffenbauer, I., and Wakeland, E.K. 1999. Genetic dissection of SLE pathogenesis: adoptive transfer of Sle 1 mediates the loss of tolerance by bone marrow-derived B cells. J. Immunol. 162:2415-2421.

24. Zhu, J., et al. 2005. T cell hyperactivity in lupus as a consequence of hyperstimulatory antigen-presenting cells. J. Clin. Invest. 115:1869-1878. doi:10.1172/ JCI23049.

25. Wakui, M., Morel, L., Butfiloski, E.J., Kim, C., and Sobel, E.S. 2005. Genetic dissection of systemic lupus erythematosus pathogenesis: partial functional complementation between Sle 1 and Sle $3 / 5$ demonstrates requirement for intracellular coexpression for full phenotypic expression of lupus. J. Immunol. 175:1337-1345.

26. Gatenby, P.A., and Irvine, M. 1994. The bcl-2 protooncogene is overexpressed in systemic lupus erythematosus. J. Autoimmun. 7:623-631.

27. Miret, C., et al. 2001. Relationship of oncogenes (sFas, Bcl-2) and cytokines (IL-10, alfa-TNF) with the activity of systemic lupus erythematosus. Anticancer Res. 21:3053-3059.

28. Graninger, W.B., Steiner, C.W., Graninger, M.T., Aringer, M., and Smolen, J.S. 2000. Cytokine regulation of apoptosis and Bcl-2 expression in lymphocytes of patients with systemic lupus erythe- 
matosus. Cell Death Differ. 7:966-972.

29. Anzelon, A.N., Wu, H., and Rickert, R.C. 2003. Pten inactivation alters peripheral B lymphocyte fate and reconstitutes CD19 function. Nat. Immunol. 4:287-294.

30. Barber, D.F., et al. 2006. Class IB-phosphatidylinositol 3-kinase (PI3K) deficiency ameliorates IAPI3K-induced systemic lupus but not $\mathrm{T}$ cell invasion. J. Immunol. 176:589-593.

31. Shaw, R.J., and Cantley, L.C. 2006. Ras, PI(3)K and mTOR signaling controls tumour cell growth. Nature. 441:424-430.

32. Sarbassov, D.D., Guertin, D.A., Ali, S.M., and Sabatini, D.M. 2005. Phosphorylation and regulation of AKT/PKB by the rictor/mTOR complex. Science. 307:1098-1101.

33. Liu, K., Liang, C., Liang, Z., Tus, K., and Wakeland, E.K. 2005. Sle1ab mediates the aberrant activation of STAT3 and Ras-ERK signaling pathways in B lymphocytes. J. Immunol. 174:1630-1637.

34. Roux, P.P., and Blenis, J. 2004. ERK and P38 MAPKactivated protein kinases: a family of protein kinases with diverse biological functions. Microbiol. Mol. Biol. Rev. 68:320-344.

35. Niculescu, F., et al. 2003. Pathogenic T cells in murine lupus exhibit spontaneous signaling activity through phosphatidylinositol 3-kinase and mitogen-activated protein kinase pathways. Arthritis Rheum. 48:1071-1079.

36. Rapoport, M.J., et al. 2002. Constitutive up-regulated activity of MAP kinase is associated with downregulated early $\mathrm{p} 21$ Ras pathway in lymphocytes of SLE patients. J. Autoimmun. 19:63-70.

37. Yi, Y., McNerney, M., and Datta, S.K. 2000. Regulatory defects in $\mathrm{Cb} 1$ and mitogen-activated protein kinase (extracellular signal-related kinase) pathways cause persistent hyperexpression of CD40 ligand in human lupus T cells. J. Immunol. 165:6627-6634.

38. Deng, C., et al. 2001. Decreased Ras-mitogen-activated protein kinase signaling may cause DNA hypometylation in $\mathrm{T}$ lymphocytes from lupus patients. Arthritis Rheum. 44:397-407.

39. Jacob, C.O., et al. 2003. Pivotal role of Stat 4 and Stat 6 in the pathogenesis of the lupus-like disease in the New Zealand mixed 2328 mice. J. Immunol. 171:1564-1571.

40. Wong, H.K., Kammer, G.M., Dennis, G., and Tsokos, G.C. 1999. Abnormal NF-kappa B activity in T lymphocytes from patients with systemic lupus erythematosus is associated with decreased p65-RelA protein expression. J. Immunol. 163:1682-1689.

41. McDonnell, T.J., et al. 1989. bcl-2 immunoglobulin transgenic mice demonstrate extended B-cell survival and follicular lymphoproliferation. Cell. 57:79-88.

42. Kuo, P., Bynoe, M.S., Wang, C., and Diamond, B. 1999. Bcl-2 leads to expression of anti-DNA B cells but no nephritis: a model for a clinical subset. Eur. J. Immunol. 29:3168-3178.

43. Miret, C., et al. 1999. Bcl-2 oncogene (B cell lymphoma/leukemia-2) levels correlate with systemic lupus erythematosus disease activity. Anticancer Res. 19:3073-3076.

44. Datta, S.R., et al. 1997. Akt phosphorylation of BAD couples survival signals to the cell-intrinsic death machinery. Cell. 91:231-241.

45. del Peso, L., Gonzalez-Garcia, M., Page, C., Herrera, R., and Nunez, G. 1997. Interleukin-3 induced phosphorylation of BAD through the protein kinase Akt. Science. 278:687-689

46. Sherr, C.J., and Roberts, J.M. 1999. CDK inhibitors: positive and negative regulators of G1-phase progression. Genes Dev. 13:1501-1512.

47. Goulvestre, C., et al. 2005. A mimic of p21WAF1/ CIP1 ameliorates murine lupus. J. Immunol. 175:6959-6967.

48. Luo, J., Maning, B.D., and Cantley, L.C. 2003. Targeting the PI3K-Akt pathway in human cancer: rationale and promise. Cancer Cell. 4:257-262.

49. Faivre, S., Kroemer, G., and Raymond, E. 2006. Current development of mTOR inhibitors as anticancer agents. Nat. Rev. Drug Discov. 5:671-688.

50. Hay, N. 2005. The Akt-mTOR tango and its relevance to cancer. Cancer Cell. 8:179-183.

51. Wullschleger, S., Loewith, R., and Hall, M.N. 2006. TOR signaling in growth and metabolism. Cell. 124:471-484

52. Sawyers, C.L. 2003. Will mTOR inhibitors make it as cancer drugs? Cancer Cell. 4:343-348.
53. Chan, S., et al. 2005. Phase II study of temsirolimus (CCI-779), a novel inhibitor of mTOR, in heavily pretreated patients with locally advanced or metastatic breast cancer. J. Clin. Oncol. 23:5314-5322.

54. Galanis, E., et al. 2005. Phase II trial of temsirolimus (CCI-779) in recurrent glioblastoma multiforme: a North Central Cancer Treatment Group Study. J. Clin. Oncol. 23:5294-5304.

55. Atkins, M.B., et al. 2004. Randomized phase II study of multiple dose levels of CCI-779, a novel mammalian target of rapamycin kinase inhibitor, in patients with advanced refractory renal cell carcinoma. J. Clin. Oncol. 22:909-918.

56. Jacinto, E., et al. 2004. Mammalian TOR complex 2 controls the actin cytoskeleton and is rapamycin insensitive. Nat. Cell Biol. 6:1122-1128.

57. Kim, D.H., et al. 2003. mTOR interacts with raptor to form a nutrient-sensitive complex that signals to the cell growth machinery. Cell. 110:163-175.

58. Giles, F.J., and Albitar, M. 2005. Mammalian target of rapamycin as a therapeutic target in leukemia. Curr. Mol. Med. 5:653-661.

59. Jundt, F., et al. 2005. A rapamycin derivative (everolimus) controls proliferation through down-regulation of truncated CCAAT enhancer binding protein $\beta$ and NF- $\mathrm{KB}$ activity in Hodgkin and anaplastic large cell lymphomas. Blood. 106:1801-1807.

60. Kumar, K.R., et al. 2006. Regulation of B cell tolerance by the lupus susceptibility gene Ly108. Science. 312:1665-1669.

61. Hackstein, H., et al. 2003. Rapamycin inhibits IL-4induced dendritic cell mobilization and function in vivo. Blood. 101:4457-4463.

62. Abe, M., and Thomson, A.W. 2003. Influence of immunosuppressive drugs on dendritic cells. Transpl. Immunol. 11:357-365.

63. Morel, L., Rudofsky, U.H., Longmate, J.A., Schiffenbauer, J., and Wakeland, E.K. 1994. Polygenic control of susceptibility to murine systemic lupus erythematosus. Immunity. 1:219-229.

64. Xie, C., Zhou, X.J., Liu, X., and Mohan, C. 2003. Enhanced susceptibility to end-organ disease in the lupus-facilitating NZW mouse strain. Artbritis Rheum. 48:1080-1092. 